\title{
How to improve drug compliance in hypertensive patients
}

Correspondence:

Dr Abdul Rasheed Khan

MBBS; MD; MESC; FACP

Head of Cardiology

Abbasi Shaheed Hospital

Karachi Medical \& Dental College

Several recent, long-term outcome studies have clearly demonstrated the benefit of blood pressure reduction on reducing cardiovascular disease. These studies have shown that lower blood pressures are associated with greater reductions in cardiovascular disease, particularly in diabetic patients.

Despite these findings, studies from the National Health and Nutritional Examination Survey (NHANES) and from the World Health Organization have indicated "that less than one quarter of hypertensive patients worldwide are controlled for high blood pressure at the goal of $140 / 90 \mathrm{~mm}$ $\mathrm{Hg}$.

Inadequately controlled hypertension remains a risk factor for coronary artery disease. These poor blood pressure control rates may contribute to the disappointing reductions in coronary artery disease and the high incidence of congestive heart failure seen among hypertensive patients.

The increasing incidence of end-stage renal failure seen among hypertensive patients may also be related to inadequately controlled hypertension.
Obtaining optimal blood pressure control in hypertensive patients remains the most important issue in the management of hypertension. Despite the fact that there are more than one hundred drugs for the treatment of hypertension and that millions of Rupees are spent on the treatment, blood pressure control is achieved in less than one quarter of hypertensive patients.

There are multiple reasons for these poor blood pressure control rates, but one of the most important remains patient compliance. A study performed in a group of patients who received free medical care in California showed that, "after 1 year on antihypertensive treatment, less than one third of the patients were still taking their antihypertensive drugs, irrespective of the class of drug with which they were treated."

In another survey of 37,643 patients with hypertension performed between October, 1992 and September, 1993 in the United Kingdom, it was reported that "change of treatment or discontinuation of treatment occurred in $40 \%-50 \%$ of the patients within 6 months."

Patient adherence to therapy must be improved 
if we are to impact blood pressure control. Patient compliance or adherence has been a major problem in the management of hypertension for as long as we have been treating this disease process.

Although the development of drugs with more favorable side-effect profiles as well as the development of once-a-day agents has resulted in some improvement in patient compliance, it still remains an important issue in the management of hypertension.

Physicians should refocus on issues that influence compliance in their management of this disease process. Many of the important issues influencing compliance can be corrected with the appropriate approach. Clearly the selection of well-tolerated drugs that can be dosed once daily is critical.

The use of low-dose combination therapy as firstline treatment for a significant percentage of hypertensive patients may represent an important change in our management and may improve compliance rates.

Achieving more rapid blood pressure control may also have some beneficial effects on patient compliance. In order to improve on the outcome in hypertensive patients, we will have to significantly improve patient, as well as physician, compliance. 